\title{
Adhesion of lymphocytes to hepatic endothelium
}

\author{
P F Lalor, D H Adams
}

\begin{abstract}
Chronic inflammation occurs when factors that regulate the process of leucocyte recruitment are disrupted, and it is dependent on recruitment, activation, and retention of lymphocytes within tissue microenvironments. The molecular mechanisms that mediate lymphocyte adhesion to vascular endothelial cells have been described by several groups, but the signals involved in the recruitment of lymphocytes via the hepatic circulation have yet to be elucidated fully. This article considers the liver as a model of organ specific lymphocyte recruitment. In this context, the roles of leucocyte and endothelial adhesion molecules and chemokines in lymphocyte recruitment are discussed. The article also reviews the mechanisms that regulate lymphocyte recirculation to the liver under both physiological and pathological conditions and draws parallels with other organs such as the gut and skin.

(F Clin Pathol: Mol Pathol 1999;52:214-219)
\end{abstract}

Keywords: chronic inflammation; liver; chemokines; lymphocyte recruitment

A complex system of lymphocyte trafficking has evolved to provide immune surveillance and to allow the immune system to recognise and respond rapidly to foreign antigen wherever it enters the body. ${ }^{1}$ When the factors that regulate this process are lost or disrupted, leucocyte recruitment continues inappropriately and chronic inflammation ensues. ${ }^{23}$ The establishment of chronic inflammation depends upon the recruitment, retention, and activation of lymphocytes in local microenvironments. In recent years, our understanding of the molecular regulation of lymphocyte recruitment has increased greatly, and these insights have important implications for disease pathogenesis and for the development of new treatments. Here, we use the liver as an example of organ specific lymphocyte recruitment to discuss the principles that govern lymphocyte recirculation and recruitment to inflammatory sites. Although concentrating on the liver, we shall draw parallels with other organs such as the gut and skin, where tissue specific lymphocyte homing has been demonstrated, to underlie the important principles of lymphocyte homing.

The liver, which has a large resident population of leucocytes, is further infiltrated by lymphocytes in response to infection or injury, although the molecular mechanisms that control these processes have yet to be elucidated fully. ${ }^{34}$ Most diseases of the liver are mediated by inflammatory and immune mechanisms. These include not only the viral hepatitides and autoimmune liver diseases, such as primary biliary cirrhosis and autoimmune hepatitis, but also toxic liver diseases such as Wilson's disease and alcoholic liver disease, where liver damage is associated with a lymphocyte rich inflammatory infiltrate..$^{5-8}$ Furthermore, the liver is an important site of exposure to foreign antigens, particularly those entering through the gut via the portal vein, and thus needs to be able to respond rapidly and selectively to harmful pathogens. Therefore, it seems likely that the resident lymphocytes within normal liver represent a recirculating memory $\mathrm{T}$ cell population that provides ongoing immune surveillance.

The molecular mechanisms that control the recruitment of lymphocytes via the hepatic circulation, under both physiological and pathological conditions, have yet to be elucidated fully. Recruitment from the circulation into the tissue is dependent on the ability of circulating lymphocytes to recognise and bind to molecules on hepatic endothelial cells that promote adhesion and subsequent transendothelial migration of lymphocytes into the liver parenchyma. ${ }^{9}$ Other liver cell types, such as Kupffer cells ${ }^{10}$ and hepatocytes, ${ }^{11}$ that can support lymphocyte adhesion or secrete cytokines to augment the local inflammatory response will also affect this process.

The paradigm of leucocyte adhesion to vascular endothelium, as described by several groups in the past few years, is likely to be applicable to the liver, although the details of the signals involved will be different. In the generally accepted model, ${ }^{12}{ }^{13}$ tethering or rolling receptors expressed on endothelial cells capture free flowing leucocytes. These receptors may be members either of the selectin family of adhesion molecules ${ }^{14}$ or the immunoglobulin superfamily. ${ }^{16-18}$ Members of both families that are involved in tethering exhibit rapid functional kinetics, which permit capture of a fast flowing cell. Once captured, the leucocyte can receive activating messages presented by endothelial cells. These messages are usually in the form of chemotactic cytokines or "chemokines", which bind to specific G protein coupled receptors on the leucocyte surface. ${ }^{1920}$ The occupancy of these receptors induces a cascade of intracellular signalling events, which results in the presentation of high affinity integrin receptors on the leucocyte surface. ${ }^{21}$ These activated integrins bind competently to their immunoglobulin family counter-receptors expressed on the endothelial surface to promote arrest and firm adhesion of the leucocyte to the vessel wall. In the presence of the appropriate migratory
Accepted for publication 4 May 1999 
$\begin{array}{llll}\text { (1) Primary adhesion (2) Triggering (3) Arrest (4) Transendothelial } & \end{array}$

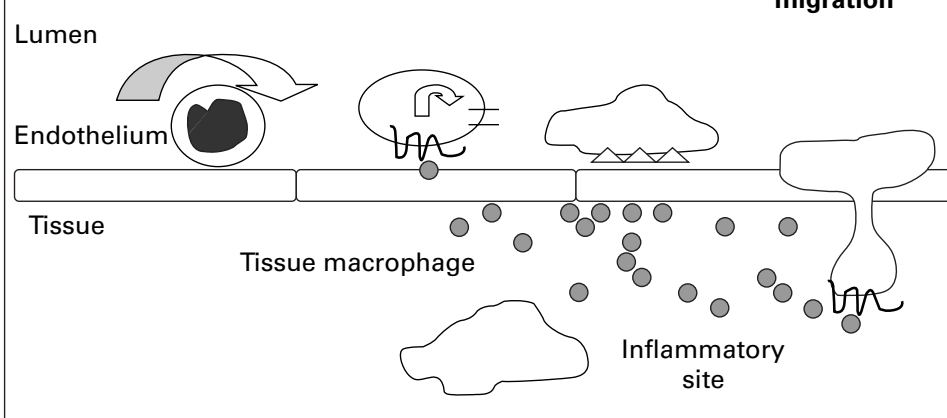

Figure 1 Free flowing leucocytes in the circulation are captured by tethering receptors (usually carbohydrate dependent) expressed on endothelial cells (1). Chemokine signals localised to the lumenal side of the endothelial surface are detected by specific, $G$ protein linked receptors expressed on the leucocyte (2). This results in a conformational change in leucocyte integrin molecules, which are converted to a high affinity state, permitting firm adhesion to endothelial expressed immunoglobulin adhesion molecules (3). Chemokine recognition also results in cytoskeletal reorganisation within the adherent leucocyte, which facilitates migration across the endothelial monolayer and into the tissue (4). Once within the tissue the leucocyte follows a chemotactic gradient of chemokine signal towards the site of inflammation. signals the leucocyte will then undergo transendothelial migration into the tissue $^{22}$ (fig 1).

Adhesion of lymphocytes to endothelial cells within different tissues appears to follow this paradigm, under both physiological and pathological conditions. Furthermore, subsets of memory lymphocytes display tissue specific homing, wherein $\mathrm{T}$ cells that have been activated in secondary lymphoid tissue subsequently return to the organ drained by that lymph node, thereby increasing the efficiency of immune surveillance. ${ }^{1}$ Hence, a T cell activated in an axillary lymph node will subsequently recirculate preferentially to the skin, whereas one activated in a mesenteric node will be programmed to home to the gut. This process is facilitated by the tissue specific expression of adhesion molecules, particularly tethering adhesion molecules, and chemokines. For example, naive $\mathrm{T}$ cells are recruited almost exclusively to secondary lymphoid tissue and this recruitment is regulated by the presence of specific molecules on high endothelial venules that are recognised by naive $\mathrm{T}$ cells. These molecules include the peripheral node addressin, which binds to L-selectin on naive $T$ cells and chemokines such as secondary lymphoid tissue chemokine (SLC) and stromal cell derived factor 1 (SDF-1) that bind to receptors preferentially expressed on naive $\mathrm{T}$ cells. ${ }^{23-27}$ Memory cells show different patterns of recirculation. For example, the migration of lymphocytes through the gut is mediated by an endothelial adhesion molecule, MAdCAM-1 (mucosal addressin cell adhesion molecule 1), that is largely restricted to mucosal vessels and which binds a ligand, $\alpha 4 \beta 1$, expressed on $T$ cells that display gut tropism. ${ }^{28} \mathrm{~A}$ further level of selectivity is provided by the fact that integrin dependent adhesion of lymphocytes to high endothelial venules in Peyer's patches is triggered by binding of the chemokine SLC (6C kine) to its receptor CCR7 on lymphocytes. ${ }^{22}$ Different

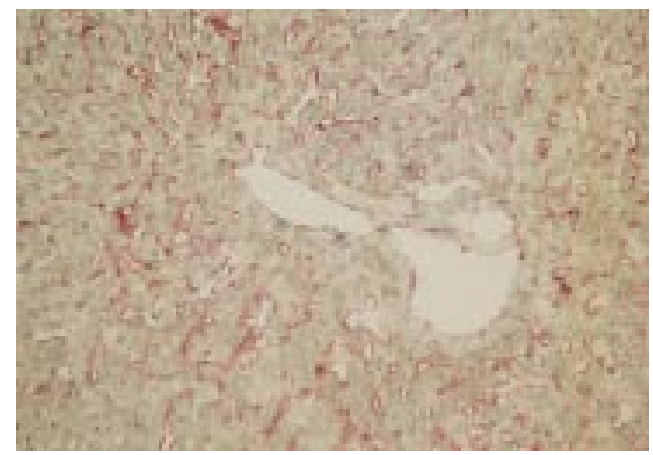

Figure 2 Expression of vascular adhesion protein 1 (VAP-1) in normal human liver. Immunohistochemical staining of a section of normal donor human liver revealing the constitutive expression of VAP-1 (stained pink) on both vascular and sinusoidal endothelial cells.

signals regulate the recruitment of memory cells to other tissues, such as skin or lung, and memory $\mathrm{T}$ cells express a distinct complement of adhesion receptors that determine where they will migrate to. Thus, the patterns of lymphocyte recirculation will depend upon the combinations of molecules expressed on the lymphocyte and the "addressins" or molecules that provide tissue with a unique molecular address.

The addressins and chemokines, which govern lymphocyte adhesion to hepatic endothelium, have yet to be defined clearly, but a review of the current understanding of this process follows.

\section{Tethering of lymphocytes by hepatic endothelium}

In most circumstances, hepatic sinusoidal endothelium fails to express members of the selectin family of adhesion receptors. ${ }^{29}$ Moreover, functional studies have shown minimal roles for $\mathrm{E}$ selectin, ${ }^{30}{ }^{31} \mathrm{P}$ selectin, and $\mathrm{L}$ selectin $^{32}$ in lymphocyte recruitment to the liver sinusoids. However, the recruitment of neutrophils to liver tissue after ischaemia has been shown to be selectin dependent, ${ }^{31} 3334$ although this recruitment may be via the portal vascular endothelium rather than directly through sinusoids. In the absence of selectin interactions, other tethering molecules are required to capture lymphocytes on hepatic endothelium. One likely candidate is vascular adhesion protein 1 (VAP-1), a homodimeric, class two transmembrane protein, ${ }^{35}{ }^{36}$ which has been shown to mediate lymphocyte adhesion to high endothelial venules in lymph nodes. ${ }^{37}$ We have shown that VAP-1 also supports lymphocyte adhesion to hepatic endothelium, both in tissue binding assays and using isolated human sinusoidal endothelium. ${ }^{38}{ }^{39}$ Hepatic endothelium is one of the few sites where this molecule is expressed constitutively (fig 2). VAP-1 mediates sialic acid dependent adhesion to hepatic endothelium under shear stress in vitro, ${ }^{38}$ and has been shown to support rolling on mesenteric vessels in vivo, ${ }^{40}$ suggesting that it functions as a rolling receptor.

Thus, VAP-1 may provide the tethering interaction between lymphocytes and hepatic sinusoidal endothelium in the absence of 


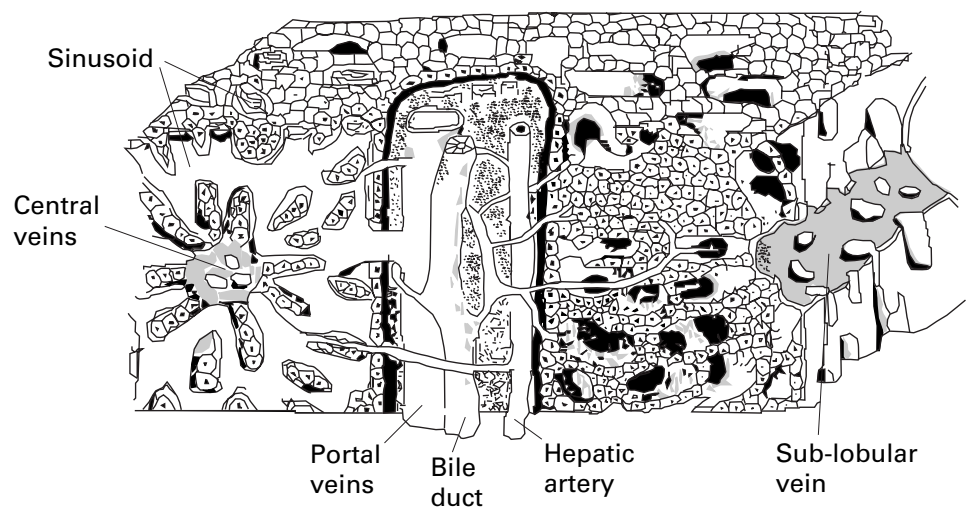

Figure 3 Schematic representation of the structure of the normal human liver lobule (adapted from Sherlock and Dooley ${ }^{45}$, with permission). The sinusoidal vessels have a typical diameter of 5-10 $\mu \mathrm{m}$ and the blood within these vessels flows at a shear stress range of $\sim 0.05$ to $0.01 \mathrm{~Pa}$. The central veins have a mean diameter of $\sim 25-30 \mu \mathrm{m}$ and the shear stress within these vessels is typically $\sim 0.2 \mathrm{~Pa}$ or higher.

selectins. Its constitutive expression in the liver suggests that it could function as an addressin to direct the recruitment of specific subsets of lymphocytes that express as yet undefined receptor/s for VAP-1. Interestingly, the highest circulating concentrations of VAP-1 are found in liver disease, further supporting its unique role in the liver. ${ }^{41}$

VAP-1 is not the only molecule that can mediate the tethering of lymphocytes to hepatic endothelium. Vascular cell adhesion molecule 1 (VCAM-1), which is induced on sinusoidal endothelium in inflammatory liver disease, can function as a tethering receptor for the capture of flowing lymphocytes. ${ }^{1628}$ VCAM-1 functions particularly well in conditions of low shear stress or where high densities of the receptor are expressed. ${ }^{18}$ Hepatic endothelium expresses low amounts of VCAM-1 under basal conditions, but these are increased considerably during inflammation. ${ }^{29} 4243$ VCAM-1 supports lymphocyte binding to hepatic endothelium in tissue binding assays. Furthermore, its ability to function well under the conditions of low shear stress that are present within the sinusoid ${ }^{44}$ (fig 3 ) supports its potential to mediate lymphocyte capture within the inflamed liver. In this respect, VCAM-1 has been shown to mediate lymphocyte ${ }^{46}$ and melanoma cell ${ }^{47}$ adhesion to hepatic endothelium.

Other molecules may also mediate tethering in inflammation. We have unpublished data demonstrating that MAdCAM 1 is induced on hepatic endothelium in certain inflammatory diseases, where it supports lymphocyte binding (AJ Grant et al, 1999, unpublished). MAdCAM-1 is a member of the immunoglobulin superfamily, and functions as an addressin for lymphocyte recirculation to mucosal tissues. It is expressed on Peyer's patch high endothelial venule endothelium and in venules in the intestinal lamina propria. $^{48}$ The presence of MAdCAM-1 on inflamed liver endothelium suggests that in some chronic inflammatory diseases the liver can be induced to express gut homing receptors. This is paralleled by the observations that VAP-1 is upregulated on inflamed mesenteric vessels. ${ }^{49}$ Thus, under certain circumstances, the gut and liver may share common addressins, suggesting the possibility of an enterohepatic recirculation of lymphocytes. Furthermore, we have used a monoclonal antibody developed in our laboratory against liver endothelium for the partial characterisation of a putative novel adhesion molecule (PF Lalor et al, 1998, unpublished). The antigen recognised by this monoclonal antibody is expressed on normal hepatic endothelial cells, particularly sinusoidal endothelium, and is upregulated under inflammatory conditions. ${ }^{50}$ We have demonstrated recently that it supports the adhesion of lymphocytes to hepatic endothelial cells under conditions of flow and we are currently defining its identity in more detail.

Firm adhesion of lymphocytes on hepatic endothelium: a requirement for chemokine mediated signalling

The activation of a family of rhodopsin-like $G$ protein linked seven transmembrane spanning receptors on the leucocyte surface is required to convert rolling adhesion to arrest. ${ }^{22} 51$ The activation of these receptors by ligand results in signalling through the $\mathrm{G}_{\mathrm{i} \alpha}$ subunit of a heterotrimeric GTP binding protein and activation of the actin cytoskeleton and cell surface integrins. The most important integrin activating factors for leucocytes are a family of chemotactic cytokines called chemokines. The interaction of chemokines with their specific receptors results in the activation of $\beta 1$ integrins and $\beta 2$ integrins and the induction of migration. This promotes arrest and firm adhesion to the vessel wall and subsequent migration across the endothelium, through the extracellular matrix, to the site of inflammation. ${ }^{19}{ }^{20} 52-54$ The ability of chemokines to bind to proteoglycans allows them to be retained in the endothelial glycocalyx and the extracellular matrix, providing a mechanism for their immobilisation at sites of inflammation. ${ }^{2155}$ Thus, lymphocytes will only be recruited to and retained in tissue if they express receptors that allow them to respond to locally presented chemokines. ${ }^{56}$

On the basis of their structure, chemokines can be classified into four groups, of which the largest subgroups are the CXC and CC families. These are defined by the presence (CXC) or absence (CC) of an amino acid between the first two cysteine residues in a conserved four cysteine motif. ${ }^{54}{ }^{57}$ Although most CXC chemokines, of which interleukin 8 (IL-8) is the best known, contain a glutamic acid-leucine-arginine (ELR) sequence near the $\mathrm{N}$-terminal and are potent chemoattractants for neutrophils, ${ }^{58}$ three CXC chemokines, IP-10 (interferon inducible protein 10), MIG (monokine induced by interferon $\gamma$ ), and I-Tac (interferon inducible $\mathrm{T}$ cell $\alpha$ chemoattractant), do not contain the ELR motif and are potent lymphocyte chemotactic factors. ${ }^{59-61}$ The CC family includes macrophage chemoattractant protein 1 (MCP-1), macrophage inflammatory protein $1 \alpha(\mathrm{MIP}-1 \alpha), \mathrm{MIP}-1 \beta$, RANTES and SLC, all of which have chemotactic activity for $\mathrm{T}$ cells in vitro. ${ }^{52}$ The recent discovery of the specific $G$ protein coupled 
receptors for many of the chemokines is enabling their roles in particular diseases to be determined. ${ }^{62}{ }^{63}$ Interactions between specific chemokines in tissue and their receptors on $\mathrm{T}$ cells may regulate the selective recruitment of lymphocytes to the liver. Several studies have reported increased expression of multiple chemokines in the inflamed liver. These include chemokines that promote $\mathrm{T}$ cell migration, such as the CC chemokines MIP$1 \alpha$, MIP-1 $\beta$, MCP-1, and RANTES and the CXC chemokines IP-10 and MIG. ${ }^{64-68}$ Furthermore, liver infiltrating $\mathrm{T}$ cells express the receptors for these chemokines ( $\mathrm{P}$ Shields and D Adams, 1998, unpublished).

However, trafficking of lymphocytes through the liver under normal conditions would require the expression of chemokines in the absence of inflammation. Some of the chemokines mentioned above, including MIP- $1 \alpha$ and RANTES, are detected in non-inflamed liver. ${ }^{65}$ In addition, several other chemokines are expressed constitutively in the liver, including the human CC chemokine liver expressed chemokine (LEC) and LARC (liver and activation regulated chemokine). ${ }^{66} 69$ Another CC chemokine, MIP-5, is restricted to the liver and gut and displays chemotactic ability for monocytes and $\mathrm{T}$ cells. ${ }^{70}$ Further information about the functions of these molecules is required to assess their roles in liver specific recruitment of lymphocytes.

Once a chemokine receptor on a lymphocyte has been activated, intracellular signalling events lead to the expression of functionally competent integrins that can bind their immunoglobulin counterparts on endothelial cells. One immunoglobulin receptor of particular importance is intercellular adhesion molecule 1 (ICAM-1), which is expressed abundantly and constitutively on both vascular and sinusoidal endothelial cells within the liver. ${ }^{71}{ }^{72}$ ICAM-1 expression is raised in response to inflammatory cytokines in vitro ${ }^{73}$ and in response to liver damage in vivo. ${ }^{74}$ ICAM-1 has been shown to mediate firm adhesion and transmigration of neutrophils ${ }^{75} 76$ and more recently $\mathrm{T}$ cells to hepatic endothelial cells in vitro. ${ }^{38}$ VCAM-1 is also expressed by activated hepatic endothelium in vivo and in vitro. Tissue binding studies demonstrate that VCAM-1 can support lymphocyte binding to liver endothelium and recent unpublished work from our group suggests it can mediate both rolling and firm adhesion to human hepatic endothelium under flow (P Lalor and D Adams, 1999, unpublished).

Once captured by, and firmly adherent to hepatic endothelium, a lymphocyte must breach the endothelial barrier and migrate into the tissue. Lymphocyte transit through vascular endothelium may be facilitated by expression of CD31, which is also used by neutrophils to migrate into the liver. ${ }^{77} 78$ It is likely that transendothelial migration involves lymphocyte passage through interendothelial tight junctions. ${ }^{79}$ However, hepatic sinusoidal endothelial cells form a discontinuous barrier without tight junctions and, there- fore, there may be a reduced reliance on factors such as CD31. To date, few studies have investigated whether lymphocyte transmigration through the architecturally and phenotypically unique sinusoidal endothelium involves novel mechanisms of transendothelial migration.

In summary, a multistep adhesion cascade similar to that proposed for vascular endothelium appears to mediate lymphocyte adhesion to hepatic endothelial cells. However, the liver demonstrates tissue specificity with respect to the combinations of adhesion molecules expressed and chemokine signals presented. These observations provide further support for the concept of tissue specific homing of lymphocytes, in which subsets of activated $\mathrm{T}$ cells display tissue tropism and recirculate to the tissue in which they were originally activated. Future research on the specific signals that regulate this process will shed light on molecules involved in tissue specific lymphocyte adhesion, and on the mechanisms of lymphocyte transmigration through specialised endothelium, such as the hepatic sinusoid. This should enable the development of new treatments for inflammatory diseases, in which the recruitment of lymphocytes to a particular tissue can be inhibited selectively without preventing normal leucocyte recruitment elsewhere. In addition, there is potential for adoptive immunotherapy, in which lymphocytes are programmed in vitro to express particular combinations of receptors that will target their recruitment to a specific tissue after reinfusion in vivo.

1 Butcher EC, Picker LJ. Lymphocyte homing and homeostasis. Science 1996;272:60-6.

2 Adams DH, Lloyd AR. Chemokines: leucocyte recruitment and activation cytokines. Lancet 1997;349:490-5.

3 Jaeschke H. Cellular adhesion molecules: regulation and functional significance in the pathogenesis of liver diseases. Am F Physiol 1997;273:G602-11.

4 Crawford JM. Cellular and molecular biology of the inflamed liver. Curr Opin Gastroenterol 1997;13:175-85.

5 Galle PR, Hofmann WJ, Wlaczak H, et al. Involvement of the CD95 (APO1/FAS) receptor and ligand in liver damage. $\mathcal{A}$ Exp Med 1995;182:1223-30.

6 Chiappelli F, Kung M, Lee P, et al. Alcohol modulation of human normal T-cell activation, maturation, and migration. Alcohol Clin Exp Res 1995;19:539-44.

7 Paronetto F. Immunologic reactions in alcoholic liver disease. Semin Liver Dis 1993;13:183-95.

8 Adams DH. Leucocyte adhesion and alcoholic liver disease. Alcohol Alcohol 1994;29:249-60.

9 Jaeschke H. Chemokines, neutrophils, and inflammatory liver-injury. Shock 1996;6:403-4.

0 Furst SM, Gandolfi AJ. Interaction of lymphocytes with Kupffer cells from halothane-exposed guinea pigs. Int Arch Allergy Immunol 1997;114:46-53.

11 Rowell DL, Eckmann L, Dwinell MB, et al. Human hepatocytes express an array of proinflammatory cytokines after agonist stimulation or bacterial invasion. Am 7 Physiol 1997;273:G322-32.

12 Adams DH, Shaw S. Leucocyte endothelial interactions and regulation of leucocyte migration. Lancet 1994;343:831-6.

13 Springer TA. Traffic signals for lymphocyte recirculation and leukocyte emigration: the multistep paradigm. Cell and leukocyte emig

14 Bevilacqua MP. Endothelial-leukocyte adhesion molecules. Annu Rev Immunol 1993;11:767-84.

5 Ebnet K, Kaldjian EP, Anderson AO, et al. Orchestrated information transfer underlying leukocyte endothelial interactions. Annu Rev Immunol 1996;14:155-77.

16 Alon R, Kassner PD, Carr MW, et al. The integrin VLA-4 supports tethering and rolling in flow on VCAM-1. I Cell Biol 1995;128:1243-53.

17 Berlin C, Bargatze RF, Campbell JJ, et al. Alpha-4 integrins mediate lymphocyte attachment and rolling under physiological flow. Cell 1995;80:413-22.

18 Lalor PF, Clements JM, Pigott R, et al. Association between receptor density, cellular activation, and transformation of adhesive behavior of flowing lymphocytes binding to VCAM-1. Eur f Immunol 1997;27:1422-6. 
19 Luster AD. Chemokines - chemotactic cytokines that mediate inflammation. N Engl 7 Med 1998;338:436-45.

20 Adams DH Lloyd AR. Chemokines: leucocyte recruitment and activation cytokines. Lancet 1997;349:490-5.

21 Tanaka Y, Adams DH, Hubscher S, et al. T-cell adhesion induced by proteoglycan-immobilized cytokine MIP-1 $\beta$. Nature 1993;361:79-82.

22 Campbell JJ, Hedrick J, Zlotnick A, et al. Chemokines and the arrest of lymphocytes rolling under flow. Science 1998; 279:381-4.

23 Pachynski RK, Wu SW, Gunn MD, et al. Secondary lymphoid-tissue chemokine (SLC) stimulates integrin alpha 4 beta 7 -mediated adhesion of lymphocytes to mucosal addressin cell adhesion molecule-1 (MAdCAM-1) under flow. f Immunol 1998;161: 952-6.

24 Bargatze RF, Jutila MA, Butcher EC. Distinct roles of L-selectin and integrins alpha-4-beta-7 and LFA-1 in lymphocyte homing to peyers patch-HEV in-situ - the multistep 108 .

25 Girard JP, Springer TA. High endothelial venules (HEVs) specialized endothelium for lymphocyte migration. Immunol Today 1995;16:449-57.

26 Kunkel EJ, Ramos CL, Steeber DA, et al. The roles of L-selectin, beta 7 integrins, and P-selectin in leukocyte rolling and adhesion in high endothelial venules of Peyer's patches. F Immunol 1998;161:2449-56.

27 Michie SA, Streeter PR, Bolt PA, et al. The human peripheral lymph node vascular addressin. An inducible endotheeral lymph node vascular addressin. An inducible endothelial antigen involved

28 Berlin C, Berg EL, Briskin MJ, et al. Alpha 4 beta 7 integrin mediates binding to the mucosal vascular addressin MAdCAM-1. Cell 1993;74:185-95.

29 Steinhoff G, Behrend M, Schrader B, et al. Expression patterns of leukocyte adhesion ligand molecules on human liver endothelia-lack of ELAM-1 and CD62 inducibility on sinusoidal endothelia and distinct distribution of VCAM-1, ICAM-1, ICAM-2 and LFA-3. Am ₹ Patho 1993;142:481-8.

30 Adams DH, Hubscher SG, Fisher NC, et al. Expression of E-selectin (CD62E) and E-selectin ligands in human liver inflammation. Hepatology 1996;24:533-8.

31 Horie Y, Wolf R, Russell J, et al. Role of Kupffer cells in gut ischemia/reperfusion-induced hepatic microvascular dysischemia/reperfusion-induced hepatic microvascul
function in mice. Hepatology 1997;26:1499-1505.

32 Wong J, Johnston B, Lee SS, et al. A minimal role for selectins in the recruitment of leukocytes into the inflamed liver microvasculature. $\mathcal{F}$ Clin Invest 1997;99:2782-90.

33 Hamamoto I, Hossain MA, Mori S, et al. Impact of adhesion molecules of the selectin family on liver microcirculation at reperfusion following cold ischemia. Transplant Int 1996;9:454-60.

34 Dulkanchainun TS, Goss JA, Imagawa DK, et al. Reduction of hepatic ischemia/reperfusion injury by a soluble P-selectin glycoprotein ligand-1. Ann Surg 1998;227:83240 .

35 Salmi M, Tohka S, Berg EL, et al. Vascular adhesion protein 1 mediates lymphocyte subtype-specific, selectinindependent recognition of vascular endothelium in human lymph nodes. I Exp Med 1997;186:598-600.

36 Salmi M, Jalkanen S. Human vascular adhesion protein-1 (VAP-1) is a unique sialoglycoprotein that mediates carbohydrate-dependent binding of lymp
endothelial-cells. F Exp Med 1996;183:569-79.

37 Salmi M, Hellman J, Jalkanen S. The role of two distinct endothelial molecules, vascular adhesion protein-1 and peripheral lymph node addressin, in the binding of lymphocyte subsets to human lymph nodes. F Immunol 1998;160:5629-36.

38 Yoong KF, McNab G, Hubscher SG, et al. Vascular adhesion protein-1 and ICAM-1 support the adhesion of tumor infiltrating lymphocytes to tumor endothelium in tumor infiltrating lymphocytes to tumor endothelium in human

39 McNab G, Reeves JL, Salmi M, et al. Vascular adhesion protein-1 supports adhesion of $\mathrm{T}$ lymphocytes to hepatic endothelium. A mechanism for $\mathrm{T}$ cell recirculation to the liver? Gastroenterology 1996;110:522-8.

40 Smith DJ, Salmi M, Bono P, et al. Cloning of vascular adhesion protein 1 reveals a novel multifunctional adhesion molecule. F Exp Med 1998;188:17-27.

41 Kurkijarvi R, Adams DH, Leino R, et al. Circulating form of human vascular adhesion protein-1 (VAP-1): increased serum levels in inflammatory liver diseases. F Immunol 1998;161:1549-57.

42 Steinhoff G, Behrend M, Schrader B, et al. Intercellular immune adhesion molecules in human liver transplants: overview on expression patterns of leukocyte receptor and ligand molecules. Hepatology 1993;18:440-53.

43 Adams DH, Burra P, Hubscher SG, et al. Endothelial activation and circulating vascular adhesion molecules in alcoholic liver disease. Hepatology 1994;19:588-94.

44 Salmi M, Adams DH, Jalkanen S. Lymphocyte trafficking in the intestine and liver. Am f Physiol 1998;274:G1-6.

45 Sherlock S, Dooley J. Diseases of the liver and biliary system. Oxford: Blackwell Scientific Publishing, 1993.

46 Yoong KF, Adams DH. Interleukin-2 restores CD3 zeta chain expression but fails to generate specific cytolytic activity in tumor infiltrating lymphocytes from patients with hepatic colorectal metastases. Br f Cancer 1998;77: 1072-81.
47 Anasagasti MJ, Alvarez A, Martin JJ, et al. Sinusoidal endothelium release of hydrogen peroxide enhances very cell adherence and tumor cytotoxicity during interleukin-1 promotion of hepatic $840-6$.

48 Briskin M, Winsor-Hines D, Shyjan A, et al. Human mucosal addressin cell adhesion molecule-1 is preferentially expressed in intestinal tract and associated lymphoid tissue. Am ₹ Pathol 1997;151:97-110.

49 Salmi M, Kalimo K, Jalkanen S. Induction and function of vascular adhesion protein 1 at sites of inflammation. $\mathcal{f}$ Exp Med 1993;178:2255-60.

50 Lalor PF, Afford S, Fear J, et al. Mab SC62 recognises a putative novel adhesion molecule that supports lymphocyte adhesion to human hepatic endothelium [abstract]. Hepatology 1998;28:514A

51 Campbell JJ, Qin SX, Bacon KB, et al. Biology of chemokine and classical chemoattractant receptorsdifferential requirements for adhesion-triggering versus chemotactic responses in lymphoid-cells. F Cell Biol 1996; 134:255-66.

52 Mackay CR. Chemokine receptors and T-cell chemotaxis. $\mathcal{F}$ Exp Med 1996;184:799-802.

53 Carr MW, Alon R, Springer TA. The C-C chemokine MCP-1 differentially modulates the avidity of beta- 1 and beta-2 integrins on T-lymphocytes. Immunity 1996;4:17987.

54 Baggiolini M. Chemokines and leukocyte traffic. Nature 1998;392:565-8

55 Tanaka Y, Kimata K, Adams DH, et al. Modulation of cytokine function by proteoglycans: sophisticated models for the regulation of cellular responses to cytokines. Proc Assoc Am Physicians 1998;110:118-25.

56 Butcher EC. Leukocyte-endothelial cell recognition: three (or more) steps to specificity and diversity. Cell 1991;67: 1033-6.

57 Siveke JT, Hamann A. T helper 1 and T helper 2 cells respond differentially to chemokines. F Immunol 1998;160: $550-4$

58 Larsen CG, Anderson AO, Appella E, et al. The neutrophilactivating protein (NAP-1) is also chemotactic for T lymphocytes. Science 1989;243:1464-6.

59 Liao F, Rabin RL, Yannelli JR, et al. Human MIG chemokine-biochemical and functional-characterization. 7 Exp Med 1995;182:1301-14.

60 Taub DD, Longo DL, Murphy WJ. Human interferoninducible protein-10 induces mononuclear cell infiltration mice and promotes the migration of human T-lymphocytes into the peripheral-tissues of human peripheral-blood lymphocytes-SCID mice. Blood 1996;87: 1423-31.

61 Farber JM. HuMIG: a new human member of the chemokine family of cytokines. Biochem Biophys Res Commun 1993; 192:223-30.

62 Qin S, Rottman JB, Myers P, et al. The chemokine receptors CXCR3 and CCR 5 mark subsets of T cells associated with certain inflammatory reactions. F Clin Invest 1998;101: 746-54.

63 Loetscher P, Uguccioni M, Bordoli L, et al. CCR5 is characteristic of Th1 lymphocytes [letter]. Nature 1998;391:344-

64 Adams DH, Fear J, Shaw S, et al. Hepatic expression of macrophage inflammatory protein-1 $\alpha$ and macrophage inflammatory protein-1 $\beta$ after liver transplantation. Transplantation 1996;61:817-25.

65 Afford SC, Fisher NC, Neil DAH, et al. Distinct patterns of chemokine expression are associated with leucocyte recruitment in alcoholic hepatitis and alcoholic cirrhosis. $\mathcal{F}$ Pathol 1998;186:82-9.

66 Shoudai K, Hieshima K, Fukuda S, et al. Isolation of cDNA encoding a novel human CC chemokine NCC-4/LEC. Biochim Biophys Acta 1998;1396:273-7.

67 Maltby J, Wright S, Bird G, et al. Chemokine levels in human liver homogenates-associations between GRO alpha and histopathological evidence of alcoholic hepatitis. Hepatology 1996;24:1156-60.

68 Narumi S, Tominaga Y, Tamaru M, et al. Expression of FN-inducible protein-10 in chronic hepatitis. F Immunol 1997;158:5536-44.

69 Hieshima K, Imai T, Opdenakker G, et al. Molecular cloning of a novel human CC chemokine, liver and activationregulated chemokine (LARC) expressed in liver. Chemoregulated chemokine (LARC) expressed in liver. Chemotactic activity for lymphocytes and gene local

70 Coulin F, Power CA, Alouani S, et al. Characterisation of macrophage inflammatory protein $-5 /$ human CC chemokine-2, a member of the macrophage-inflammatory-
protein family of chemokines. Eur $\mathscr{f}$ Biochem 1997;248: protein

71 Scoazec JY, Durand F, Degott C, et al. Expression of cytokine-dependent adhesion molecules in postreperfusion biopsy specimens of liver allografts. Gastroenterology 1994; 107:1094-102

72 Adams DH, Hubscher SG, Shaw J, et al. Increased expression of ICAM-1 on bile ducts in primary biliary cirrhosis and primary sclerosing cholangitis. Hepatology 1991; 14:426-32.

73 McNab G, Afford SC, Morland CM, et al. Cultured human hepatic sinusoidal endothelial cells express and secrete adhesion molecules and chemokines that are important for leukocyte recruitment to the liver. In: Wisse E, Knook DL, Balabaud C, eds. Cells of the hepatic sinusoid. Leiden: The Kupffer Cell Foundation, 1997:123-7. 
74 Yachida S, Kokudo Y, Wakabayashi H, et al. Morphological and functional alterations to sinusoidal endothelial cells in the early phase of endotoxin-induced liver failure after partial hepatectomy in rats. Virchows Arch 1998;433: 173-81.

75 Sakamoto S, Okanoue T, Itoh Y, et al. Intercellular adhesion molecule-1 and CD18 are involved in neutrophil adhesion and its cytotoxicity to cultured sinusoidal endothelial cells and its cytotoxicity to cultured sinusoi
in rats. Hepatology 1997;26:658-63.

76 Morland CM, Fear J, McNab G, et al. Promotion of leukocyte transendothelial cell migration by chemokines derived from human biliary epithelial cells in vitro. Proc Assoc Am Physicians 1997;109:372-82.
77 Bird IN, Spragg JH, Ager A, et al. Studies of lymphocyte transendothelial migration: analysis of migrated cell phenotypes with regard to CD31 (PECAM-1), CD45RA and CD45RO. Immunology 1993;80.553-60.

78 Chosay JG, Fisher MA, Farhood A, et al. Role of PECAM-1 (CD31) in neutrophil transmigration in murine models of liver and peritoneal inflammation. Am f Physiol 1998;37: G776-82.

79 Hill ME, Bird IN, Daniels RH, et al. Endothelial cell-associated platelet-activating-factor primes neutrophils for enhanced superoxide production and arachidonic-acid release during adhesion to but not transmigration across IL-1 $\beta$-treated endothelial monolayers. F Immunol 1994; 153:3673-83. 